\title{
A cellular automaton-based passive-acoustic technique for topological characterisation of objects in fluid with potential application to carotid artery plaques
}

\section{Matthew Allan Burley Delaney*}

Faculty of Arts, Science and Technology,

University of Northampton,

Waterside Campus,

University Drive, Northampton, NN1 5PH, UK

Email: matt.delaney2@gmail.com

*Corresponding author

\section{Gillian Pearce}

School of Mechanical Engineering,

Birmingham University,

B15 2TT, UK

Email: gpearce2011@gmail.com

\begin{abstract}
We present a system for the detection and characterisation of objects located in tubes of flowing fluid. Our system makes use of semiotic analysis of a driven Greenberg-Hastings cellular automaton. Our results indicate that this system is highly effective in both detecting and distinguishing topological features of different objects. We conclude that we have created a potentially effective and relatively low cost system with strong potential for the detection and initial analysis of plaques causing occlusions in the carotid artery. We further conclude that the resulting system could potentially be used as a front-line screening method in medical settings such as GP surgeries and clinics.
\end{abstract}

Keywords: carotid; artery; fluid-dynamics; cellular-automata; semiotics; medical-imaging.

Reference to this paper should be made as follows: Delaney, M.A.B. and Pearce, G. (2019) 'A cellular automaton-based passive-acoustic technique for topological characterisation of objects in fluid with potential application to carotid artery plaques', Int. J. Computational Medicine and Healthcare, Vol. 1, No. 1, pp.5-15.

Biographical notes: Matthew Allan Burley Delaney holds a PhD in Computer Science and Nonlinear Physics applied to Medical Imaging. He is a former Senior Lecturer and Course Leader in Computer Science and he currently works in industry where he specialises in programming language implementation.

Gillian Pearce has over 80 publications in peer reviewed journals. She graduated with a $\mathrm{PhD}$ in Astrophysics, and a degree in Medicine (B.M B.Ch) from Oxford University. She practised as a Medical Doctor before undertaking 
research in stroke medicine and more recently in cancer research. She has several medical innovations and patents, and held several research fellowships, including an Atlas Research Fellow at Oxford University. She currently holds an Honorary Research Fellowship at Birmingham University, UK.

\section{Introduction}

Atheromatous plaques may occur in the arteries of the human body, such as the carotid artery. These plaques are formed when damage occurs to the internal wall of the artery. Such damage may arise due to traumatic insult caused by e.g. carcinogens in tobacco smoke. The mechanism of formation of such plaques is well known (http://www.patient. co.uk/doctor/atherosclerosis). Over the course of time, the plaque in the artery may become larger leading to partial occlusion of the artery and subsequently blood clots may also form at the site of the plaque. If this blood clot and or material from the plaque becomes detached and passes in the blood flow to the cerebral circulation, then a stroke may occur in the brain, leading to potentially devastating consequences for that patient.

Often such plaques are not detected until there is extensive occlusion of the artery ( $\sim 70 \%$ occlusion) when symptoms become apparent such as amaurosis fugax (http:// www.gpnotebook.co.uk/simplepage.cfm?ID=-704249856) (temporary but reversible narrowing of the visual field(s) which often lasts for seconds), or a tingling/numbing sensation in an area of the body-frequently hands, which again lasts for seconds or minutes, before recovery, or similarly a temporary loss of motor power or loss of speech or memory lasting for seconds or minutes. Such symptoms are associated with the condition known as transient ischaemic attack (TIA) also called mini-stroke. It is believed that there are 35 mini-strokes per 100,000 people each year) (http://www. patient.co.uk/health/transient-ischaemic-attack). It is also recognised that many patients suffering a mini-stroke will develop a full blown stroke within one year of suffering a TIA. In the UK alone there are 150,000 strokes (http://www.stroke.org.uk/factsheet/whatis-a-stroke). A relatively straightforward operation called a carotid endarterectomy can be performed in patients who are known to have a plaque in the carotid artery. In the USA, some 140,000 carotid endarterectomy operations are performed each year (http://www. ninds.nih.gov/disorders/stroke/carotid_endarterectomy_backgrounder.htm).

Consequently, it is helpful to detect such plaques so that they can be removed. Currently, these plaques are detected by ultrasound (USS), which normally involves the patient being referred to a hospital for USS screening of the carotid arteries. The interpretation of USS images requires a trained USS technician. Clearly it would be advantageous to have a device that can detect plaques in the carotid (and other) arteries as early on as possible, before the plaque has occluded a significant percentage of the arterial diameter, increasing the changes of blood clots forming at the site of the plaque, or before it has reached the stage where parts of the plaque may become detached and travel to the brain giving rise to a stroke. It would be even more useful if such a device could be used by a wider range of people, e.g., nurses in GP surgeries, or even by the general public, as a front line screening device. Such a device needs to work by using software that has been developed to be fairly automated in its mode of operation. 
Delaney (2014) has developed such software as a PhD thesis. Previous publications relating to this automated software system have been presented in Burley et al. (2006, 2008) and Burley and Pearce (2010).

To demonstrate the efficacy of the system, trial artificial plaques were made of a material called polymorph, which has properties in common with arterial plaques. These artificial plaques were fashioned into a range of sizes and shapes (Figures 1 to 7). Rather than modelling the plaques on actual examples from patients, they were instead pragmatically designed to exhibit highly distinctive differences in topology; this facilitates reliable calibration and investigation of the system during these early stages of its development. The plaques were then sited in rubber tubing. Fluid (water) was passed through the tubing and the sound output generated when the fluid flowed over each plaque was recorded and analysed by the software. This situation is akin to the sound made by blood in the carotid artery as blood flows over the plaque that partially occludes the artery. Such sounds may be heard by doctors listening through a stethoscope placed over the artery at the site of the plaque, and these sounds are referred to as carotid bruits. The sound (the bruit or noise) generated by the blood/fluid as it flows over a plaque will depend on the morphology (size and shape) of the plaque. This sound is directly related to the output of the signal processed by the software (Figures 1 to 5).

\section{The software system used}

Burley and Pearce (2010) have shown previously that the Maclaurin power-series representation of a part of the diffusion function of the Greenberg-Hastings cellular automaton could be used to produce an algorithm (henceforth, to be referred to as a driven cellular automaton or DCA) which characterises (from the sound produced by fluid turbulence in a physical simulation of a partially occluded carotid artery) the level of occlusion present. Having established that the system produces distinct images for sound-recordings taken from arteries afflicted by different levels of occlusion, we proceeded to test its ability to respond to differences in plaque-topology.

Figure $1 \mathrm{p} 1$ - a plaque consisting of an approximately hemispherical mound (see online version for colours)

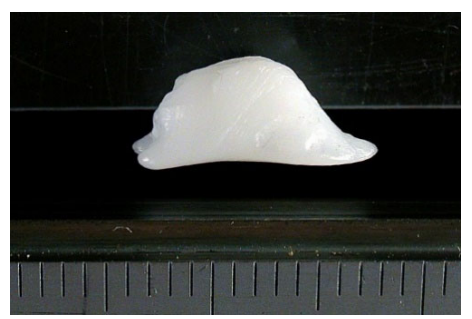

Note: Short-name: blob-mound; scale in $\mathrm{mm}$.

The images produced from the existing system described were informative for the artificial plaque-topologies tested but were not considered distinct enough to allow quick and easy analysis by a user who is not in possession of detailed knowledge of the underlying algorithms. For this reason, the two-dimensional images were treated as 
terrain-maps for three-dimensional rendering, with the third-dimension being provided by overlaying the amplitude values of the original recording on this map and using them to provide the height of the peaks. This approach increases the amount of variation between different output images.

Figure 2 p2 - a square version of the hemispherical mound (see online version for colours)

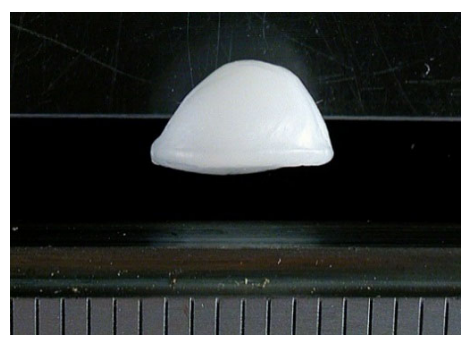

Note: Short-name: blob-square; scale in $\mathrm{mm}$.

Figure 3 p3 - a small thin ridge shape (see online version for colours)

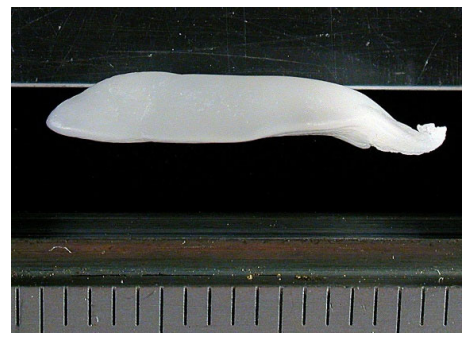

Note: Short-name: thin-ridge small; scale in $\mathrm{mm}$.

Figure 4 p4 - a more bulbous ridge shape (see online version for colours)

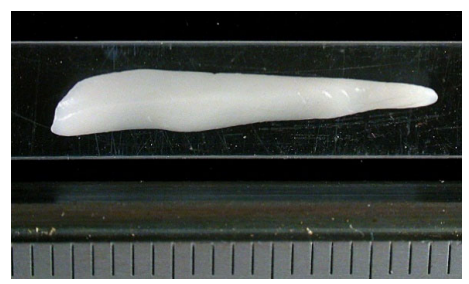

Note: Short-name: fat-ridge small; scale in $\mathrm{mm}$.

Figure 5 p5 - a larger version of fat-ridge small (see online version for colours)

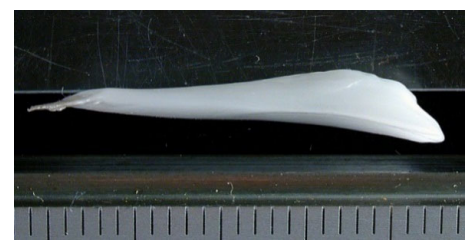

Note: Scale in $\mathrm{mm}$. 
Artificial plaques were constructed from polymorph with the following properties shown in Figures 1 to 7.

Figure 6 p6 - a small plaque with stalagmite-like protrusions (see online version for colours)

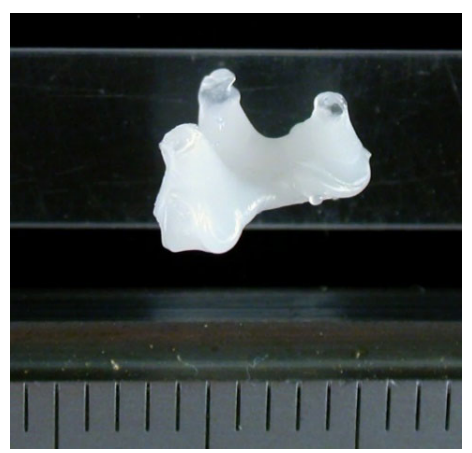

Note: Short-name: small-pokey; scale in mm.

Figure $7 \quad$ p7 - a larger version of small-pokey (see online version for colours)

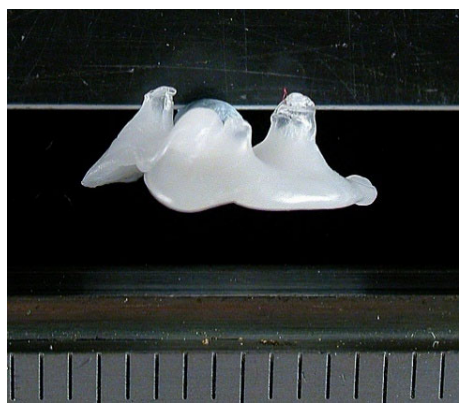

Note: Scale in $\mathrm{mm}$.

\section{Procedure}

Each plaque was situated in the simulated artery in turn. Ten second recordings were then taken allowing the resultant data being to be broken up into four sequences of amplitude-values, each 2.5 seconds in length. The imaging algorithm was run on each sequence producing four images per plaque.

Figure 8 System output for plaque p1 (see online version for colours)

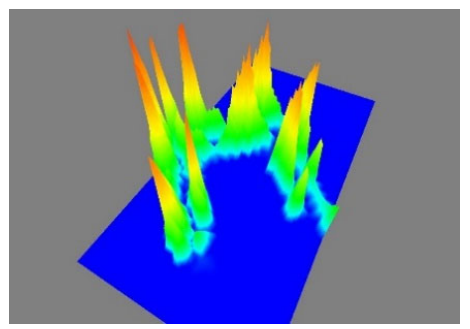


Figure 9 System output for plaque p2 (see online version for colours)

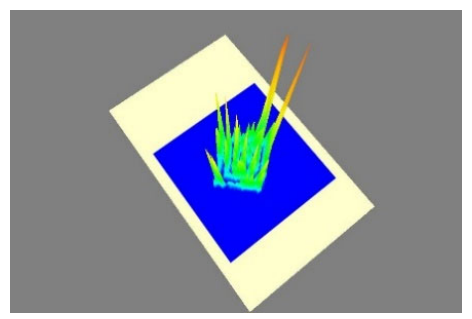

Figure 10 System output for plaque p3 (see online version for colours)

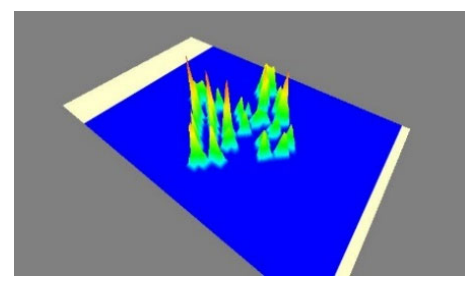

Figure 11 System output for plaque p4 (see online version for colours)

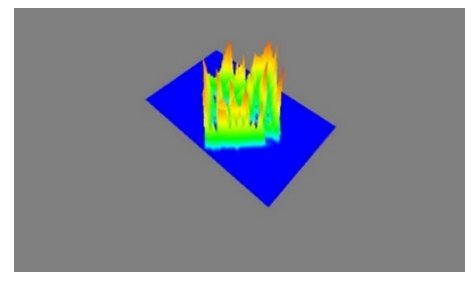

Figure 12 System output for plaque p5 (see online version for colours)

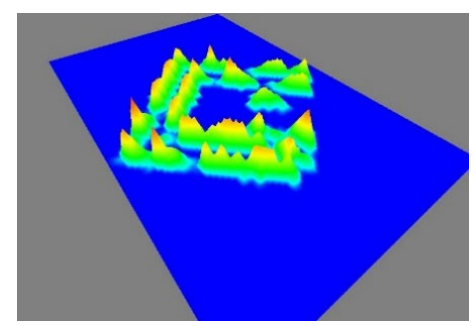

Figure 13 System output for plaque p6 (see online version for colours)

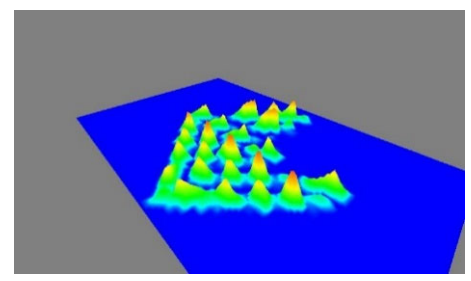


Figure 14 System output for plaque p7 (see online version for colours)

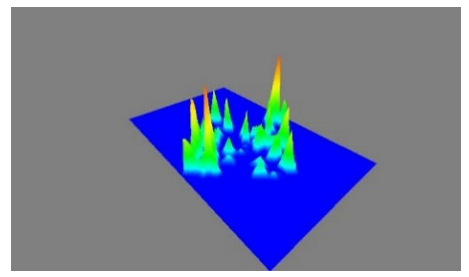

\section{Analysis}

\subsection{Application of semiotics}

The resultant images from the DCA system are only useful if it is possible to create a framework for their interpretation that can be used to train medical staff. For this aspect of the system, we make use of the discipline of semiotics: the study of signs. Originating with the philosophical analysis of concepts such as the difference between sign and signified (Chandler, 2013), the field has been considered in the medical domain by Baer (1988) and with specific focus on the analysis of X-ray images by Cantor (2000). We begin by identifying those aspects of the DCA output images that are sufficiently selfevident to be considered axioms on which to build a richer framework.

\subsection{Axiomatic basis}

It is helpful to begin by defining certain criteria that might be expected from the images produced by the system. These criteria are considered to be axiomatic in that they are self-evident, requiring only simple logical reasoning about the apparatus and the phenomenon under investigation in order to satisfy us as to their appropriateness.

1 Contrast: no two plaque topologies should produce the same image topologies.

2 Signature: minor variations are acceptable between runs but there should be an indisputable common 'essence' shared by all of the images for a given plaque-topology.

3 Scalability: the same topology in different sizes should produce similar images, ideally with only a variance in size in the image-topology.

As to the first of these criteria, it is clear that the system responds differently and distinctly for each of the topologies tested. The most significant point to draw from a comparison in this context is that the crucial aspects that result in different signatures appear to be 'base-pattern' (i.e., the tendency for certain areas of the grid to always contain peaks for a given plaque-topology) and 'inter-peak ratio' (i.e., the distribution of high and low peaks is close to constant for a given plaque-topology).

Regarding the criterion of signature, it would seem to be self-evident that for a given plaque-topology, the four runs always share enough features to allow an image from that group to be easily matched against another from the same group. 
The most useful plaque-topologies for an analysis of scalability are: fat-ridge small vs. fat-ridge large and small-pokey vs. large-pokey. These image-topologies, while not only affected by scale, do seem to indicate that the basic signature of the form is preserved between small and large versions. It is interesting to note that for the fat-ridge plaques, an increase in size corresponds to a decrease in peak-height but for the 'pokey' topology, the opposite is almost true. Intuitively, it would seem reasonable that the ridge shape, being far more uniform than a plaque consisting of several different protrusions, would result in less variance in its resultant image-topology.

It should be noted that the images displayed are not actual pictures of the simulated plaques. This is not however important within the context of roentgen semiotics as formalised by Cantor (2000) where, even in cases where a medical-imaging system produces signs intended to represent their objects in respect of their actual existence (i.e., a photograph of your friend is exactly that; it does not require interpretation to discern what it is). Diagnostic analysis is still based upon the recognition and interpretation of abstractions (for instance, in the case of the roentgen semiotics of x-ray images sharp changes in contrast and unusual distribution of light and dark areas are of far more use than treating the image as a photograph (Cantor, 2000)). It is Peirce's concept (Hoopes, 1991) of interpretant that is significant in determining the utility of the system output.

The user would require some instruction concerning what to look for (as is the case with virtually every existing medical imaging system) and we can therefore proceed to create a table of features for interpretation.

Table 1 Semiotic categories for DCA image analysis

\begin{tabular}{ll}
\hline Altitude & $\begin{array}{l}\text { The height of the peaks present in an image which has gained a topological } \\
\text { boundary. This sign is quantitative in nature. }\end{array}$ \\
$\begin{array}{l}\text { Altitudinal } \\
\text { homogeneity }\end{array}$ & $\begin{array}{l}\text { Whether or not the peaks in an image are of approximately the same height } \\
\text { or not. This is sign is possessive in nature - an image either has it or does not } \\
\text { as can be seen from the example images; there is no situation where exact } \\
\text { altitudinal homogeneity has a different meaning to slightly less altitudinal } \\
\text { homogeneity. }\end{array}$ \\
Phase regularity & $\begin{array}{l}\text { Whether or not the peaks appear at equally-spaced intervals. } \\
\text { Planar }\end{array}$ \\
completeness & $\begin{array}{l}\text { Knowing what we do about the normal shape of the patterns produced by a } \\
\text { Greenberg-Hastings cellular automaton, is there a peak (of any altitude) for } \\
\text { each cell? At the time of writing, this sign would appear to be possessive in } \\
\text { nature, at least from the point of view of what we can diagnose from it. }\end{array}$ \\
Enclosure & $\begin{array}{l}\text { Does one area of the image enclose an area composed of a different kind of } \\
\text { sign? This sign is possessive in nature. }\end{array}$ \\
\hline
\end{tabular}

Each of these signs can be gained and lost with the amount of such gain or loss only significant in the case of altitude. The images produced via physical simulation can now be analysed within this framework.

It can be seen from Table 2 that each of the plaque types analysed is distinctly and easily categorised using the semiotics properties we have defined. We have therefore provided a basis from which to train radiologists in the interpretation of DCA images.

It is important to compare the images produced by the system for the seven polymorph plaques with those produced for a simulated artery that does not contain an obstruction. These images form the control-data for the analysis and, equally importantly, allow us to see how the DCA informs us that no arterial occlusion is present. 
Table 2 Analysis of DCA output images by semiotic categories

\begin{tabular}{lccc}
\hline Image & Altitude & Altitudinal-homogeneity & Phase-regularity \\
\hline P1: blob-mound & High & No & No \\
P2: blob-square & Low & No & No \\
P3: thin-ridge small & Low & Yes & Yes \\
P4: fat-ridge small & High & Yes & No \\
P5: fat-ridge large & Low & Yes & No \\
P6: small-pokey & Low & Yes & Yes \\
P7: large-pokey & High & No & No \\
\hline Image & Planar-completeness & \multicolumn{2}{c}{ Enclosure } \\
\hline P1: blob-mound & \multicolumn{2}{c}{ Yes } \\
P2: blob-square & No & No \\
P3: thin-ridge small & Yes & \multicolumn{2}{c}{ No } \\
P4: fat-ridge small & No & \multicolumn{2}{c}{ No } \\
P5: fat-ridge large & Yes & \multicolumn{2}{c}{ Yes } \\
P6: small-pokey & No & \multicolumn{2}{c}{ Yes } \\
P7: large-pokey & No & No \\
\hline
\end{tabular}

Figure 15 System output showing fluctuation in base-image for absence of plaque (see online version for colours)

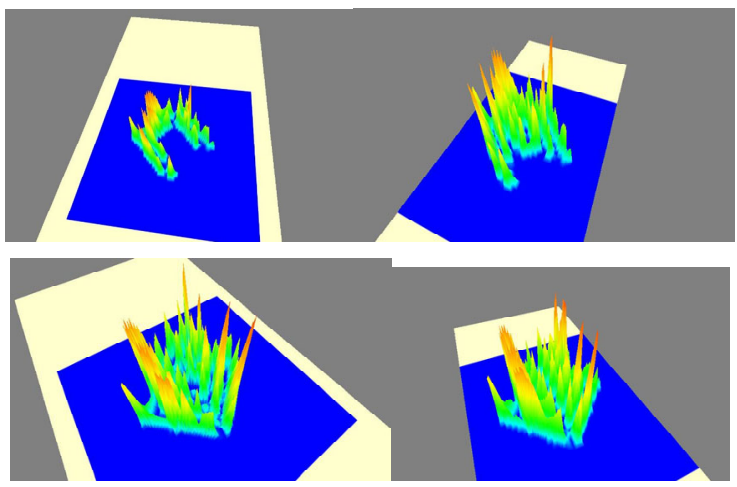

Note: Note that peak-height has been automatically scaled-up - the peaks shown would all qualify as having 'low' altitude.

The first point to take into account in this context is that the range of amplitudes divided up into buckets corresponding to the terms of the Maclaurin power series is itself computed from the input data, the idea being that the significance of fluctuation in the signal is relative to the minimum and maximum amplitude values for that particular instance of the phenomenon. For the control-recording, this range was very small but the DCA nevertheless adapted to it and produced patterns characteristic of the Greenberg-Hastings cellular automaton. Small amplitude ranges also occurred for plaques $\mathrm{p} 5$ and $\mathrm{p} 6$ and so the question arises as to how one reliably tells the difference between these two plaque topologies and the absence of a plaque. The answer comes 
from the fact that, for the control-data, there is no consistent categorisation possible given the criteria above. In general, the images have low altitude and do not exhibit altitudinal homogeneity whilst consistently exhibiting phase-regularity; this is not a categorisation seen in any of the seven polymorph plaques and might suffice to indicate the system's base-output on its own. It is, however, also the case that the presence of planarcompleteness and enclosure varies to a large extent between the base-images - something that does not occur in repeat runs for the polymorph plaques. As a result, the absence of a plaque can be reliably identified if the images from repeat-measurements defy full categorisation within the semiotic framework.

\section{Discussion}

The next phase of work for this system will be to have the images rendered continuously and in real-time as the data comes in; this would allow the absence of a plaque to be identified by an unstable image consisting only of low-altitude peaks, a stable image of any other kind indicating the presence of an occlusion. Following this, the systems' overall behaviour will need to be examined under pulsatile-flow since this will alter the nature of the sound being processed. It is expected, however, that the nature of the DCA system will place the majority of adjustments within the domain of the semiotic framework rather than the algorithm itself; this is another advantage of the abstract nature of the images produced. The next stages of our development of this system will also involve calibration against USS results for plaques of known dimensions and forms.

\section{Conclusions}

We have presented a means of characterising (via abstract 3D images and a simple framework for their interpretation) the topological features of objects placed into simulated arteries. By recognising that the interpretation of any image is, in fact, a semiotic process and necessarily abstract, we have created a system for producing direct pictorial representations of these occlusions resulting in a system that is efficient at detection without the need for specialist hardware. We believe that this system has strong potential to be developed as an affordable approach to front-line screening in low-budget medical sites such as GP surgeries and clinics.

\section{Acknowledgements}

Special thanks to Colleen Delaney for producing the artificial polymorph plaques, Malcolm Inman for the photography and David Luckhurst for rendering the system output into image-files.

Parts of the work described in this paper were undertaken as part of a $\mathrm{PhD}$ at the University of Northampton, thesis copyright Matthew Allan Burley Delaney. 


\section{References}

Baer, E. (1988) Medical Semiotics, University Press of America, Maryland.

Burley, M. and Pearce, G. (2010) 'Cellular-automaton profiling of acoustic data for feature extraction of turbulent flow in occluded carotid arteries', Computer Modeling and Simulation, International Conference on(UKSIM), pp.216-220, Cambridge, UK.

Burley, M., Bechkoum, K. and Pearce, G. (2006) 'A formative survey of geometric algebra for multivariate modelling', in European Modelling Symposium UK Society for Modelling and Simulation (2006), London, UCL, London.

Burley, M., Bechkoum, K. and Pearce, G. (2008) 'An architecture for the automatic derivation of imaging equations for the visualisation of carotid arterial plaques', in Tenth International Conference on Computer Modeling and Simulation, Emmanuel College, Cambridge University, Cambridge 1-3 April, pp.112-116.

Cantor, R.M. (2000) 'Foundations of Roentgen semiotics', Semiotica: Journal of the International Association for Semiotic Studies, Vol. 131, Nos. 1-2, pp.1-18.

Chandler, D. (2013) Semiotics for Beginners [online] http://www.aber.ac.uk/media/Documents/ S4B/semiotic.html (accessed 16 June 2013).

Delaney, M. (2014) A Cellular Automaton - Based System for the Identification of Topological Features of Carotid Artery Plaques, PhD thesis, University of Northampton, UK.

Hoopes, J. (1991) Peirce on Signs, The University of North Carolina Press, Chapel Hill, NC. 